\title{
Flow dynamics during incremental velocity running
}

\author{
Aleix Gibert ${ }^{1}$, Natàlia Balagué ${ }^{2}$, Carlota Torrents ${ }^{3}$, Gershon Tenenbaum ${ }^{4}$ \\ ${ }^{1}$ Escola Superior de Ciències de la Salut Tecnocampus (ESCST), University Pompeu Fabra (UPF), \\ Mataró, Spain \\ ${ }^{2}$ Institut Nacional d'Educació Física de Catalunya (INEFC), University of Barcelona (UB), \\ Barcelona, Spain \\ ${ }^{3}$ Institut Nacional d'Educació Física de Catalunya (INEFC), University of Lleida (UdL), Lleida, Spain \\ ${ }^{4}$ B. Ivcher School of Psychology, Interdisciplinary Center (IDC), Herzliya, Israel \\ ${ }^{1}$ Corresponding author \\ E-mail: ${ }^{1}$ aleix.gibert.buxeda@gmail.com, ${ }^{2}$ nataliabalague@gmail.com, ${ }^{3}$ carlotat@yahoo.es, \\ ${ }^{4}$ gtenenbaum@admin.fsu.edu
}

Received 31 December 2020; received in revised form 7 March 2021; accepted 14 March 2021 DOI https://doi.org/10.21595/chs.2021.21858

Check for updates

Copyright $(0) 2021$ Aleix Gibert, et al. This is an open access article distributed under the Creative Commons Attribution License, which permits unrestricted use, distribution, and reproduction in any medium, provided the original work is properly cited.

\begin{abstract}
The purpose of this study was to explore the flow dynamics during incremental velocity running performed until voluntary exhaustion. Twenty runners performed an incremental-velocity test (The Université of Montréal Track Test; UMTT) while self-reporting their "in flow" and "not in flow" experienced states. Task endurance was divided into five-time windows and flow state was plotted for each participant to determine the velocity-flow relations. Friedman ANOVA and Wilcoxon matched-pairs test were performed to follow the flow dynamic throughout the time windows. A meta-stable flow experience dynamic was revealed during the incremental running velocity test and an abrupt decrease of the "in flow" experience upon approaching voluntary exhaustion was evident. Self-monitoring flow experience dynamics can complement the physiological measures for monitoring exercise tolerance.
\end{abstract}

Keywords: flow experience, self-monitoring, metastability, phenomenological perspective.

\section{Introduction}

Running is one of the most popular and practiced physical activities for maintaining fitness. Adherence to running depends largely on the state of pleasantness and pleasure one feels while exercising $[1,2]$. Thus, self-monitoring and self-regulating the pace to maintain pleasure is recommended [3]. Some runners even report on a state called "flow", an optimal state in which complete absorption in the task at hand results in several experiential qualities [4]. The balance between the demands of the run and the aerobic capacity of the runner is considered the main determinant of the flow experience [5]. However, not every pleasant running pace results in experiencing flow (e.g., too easy pace). Moreover, flow is associated with increased intrinsic motivation, which upturns the long-term running performance [6], a goal that embraces runners of all levels.

The relation between flow and task performance has traditionally considered psychological [7] and psychophysiological factors [8]. Recently, a study has characterized flow as an embodied state, and flow experience as a property of the performer-environment coupling [9]. In fact, according to the ecological psychology, the performer and the environment are continuously integrated through the action-perception cycle [10], which is crucial for understanding how conscious experiences emerge. The information about oneself (e.g., proprioception, interoception) in relation to the environmental information is defined by a key concept for survival in ecological psychology: the informed awareness [11], which is assumed to reach the state of flow [9]. However, the relation of survival, fitness and health with the flow state is still an unexplored research topic.

The balance between the demands of the activity (i.e., the environment) and the individual's capacity to cope with this demand has been considered as a salient requirement for experiencing flow [5]. The interrelation between exercise workload and perceptual-affective responses was evident in several studies $[12,13]$. For instance, attention (effort-related attention model) [14] and 
affect (dual-mode model) [1] were found to change as a function of exercise intensity. Moreover, recent studies explored the dynamics of psychobiological variables like perceived exertion, attention focus, and pain feeling during exercise through self-monitoring and self-reporting protocols [15-18]. A noteworthy conclusion extracted from these studies is that the coupling between the runner's perceptions and the environmental demands determines the fluctuation dynamics of these psychobiological constructs during exercise. Specifically, the dynamic psychobiological model of exercise-induced fatigue framework [15] proposes a flexible and context-dependent integration between the exerciser and the environment, where components transiently couple and decouple at different intensities displaying a metastable dynamic.

The concept of metastability - the existence and evolution of systems through transient and semi-transient states [19] is not new. The spatiotemporal organization of brain activity through transient, metastable states was proposed by Kelso [20], but only recently has been experimentally identified at the phenomenological level [16, 21, 22]. At such phenomenological level, metastability is defined as a flexible integration and segregation among attentional and perceptual degrees of freedom that form the experiential states of the performer [18]. This line of investigation provides a platform for exploring the coupling and decoupling dynamics of complex goal-directed systems.

The dynamic of psychobiological properties may be brought about spontaneously by manipulation of constraints (e.g., the velocity of the run). The term "spontaneous" means that changes arise without assuming involvement of superordinate processes that explicitly impose that change [23]. Thus, a dynamic perspective on the study of flow may guide a solid understanding of how velocity and effort accumulation influence the emergence/dissolution of this state and test its hypothesized metastable behavior. This may enrich the theory and help in providing practical recommendations to practitioners of all levels who seek for the multiple benefits of experiencing a flow state.

The dynamic perspective for studying flow requires continuous self-monitoring and reporting techniques during the activity, which has already been used for capturing the dynamics of psychobiological variables such as attention focus [22], perceived exertion [21] and pain [17]. Even though being aware of flow during the activity is considered counterproductive as it inhibits one's ability to merge action and awareness [24], it can be argued that running is a cyclic and simple type of activity and is less affected by the conscious awareness of flow. Furthermore, the use of non-verbal self-report signals must not hinder the flow experience which has already been reported by elite golfers [25].

Accordingly, the purpose of this research was to study the dynamic of the "flow experience" during an incremental velocity-running test performed until voluntary exhaustion. Drawing upon a complex dynamic approach, we hypothesized a meta-stable flow experience during incremental velocity running, alternating the "in flow" and "not in flow" states, with a decrease of the "in flow" experience upon approaching voluntary exhaustion.

\section{Method}

\subsection{Participants}

Twenty Caucasian voluntary Sport Science students ( 14 males and 6 females; $M_{\text {age }}=20$ years old, $S D=4.94)$, previously familiarized with the motor and cognitive tasks of the experiment and with the required self-monitoring and self-reporting abilities, participated in the study. They had no specific running training experience but were undergoing regular aerobic exercise $(M=5.98 \mathrm{~h} /$ week, $S D=2.70)$. Exclusion criteria consisted of current or previous injury affecting the test, and any other condition that may prevent the performance of a maximal exercise protocol. 


\subsection{Intervention and procedure}

The general aims of the study were explained and discussed with the participants prior to signing the written informed consent. The experiment was approved by the local research ethical committee (Comité d'Ètica d'Investigacions clíniques de l'Administració Esportiva de Catalunya).

Running task. The Université of Montréal Track Test (UMTT) [26] was performed on a 200 $\mathrm{m}$ outdoor natural grass track with inclined curves (ratio $=16 \mathrm{~m}$ ) and pace markers (red pylons) set every $50 \mathrm{~m}$. The runners were paced using a pre-recorded tape with sound signals. The first stage was set at a walking velocity (i.e., $6.00 \mathrm{~km} \cdot \mathrm{h}^{-1}$ ); thereafter the velocity increased gradually every 2-min (see Fig. 1 for specific velocity in each stage). Participants were instructed to complete as many stages as possible. The test was terminated voluntary, and/or when participants could not reach two consecutives pylons at the required time.

Perceived demands and manipulation check. Following a 2-min cool-down period, participants were asked to rate the questions "for me, personally, the current demands of the run was..." on a Likert-type scale ranging from 1 (very low) to 9 (very high), and also "did you run as much as you could have?" and "have you reported all the changes of your flow state?" on a Likert-type scale with anchors ranging from 0 (not at all) to 10 (greatly).

Flow monitoring and reporting procedures. To record the dynamics of flow experience during the run participants were asked to self-monitor and self-report changes of their flow experience using a continuous online paradigm [27, 28], a widely applied strategy in the attention focus literature $[18,21,22]$. Examples extracted from the Spanish version of the Flow State Scale $[29,30]$ were used for making the runners familiar with the experience of flow, including, "I felt in total control of what I was doing," "I had a total concentration," and "the experience left me feeling great". Accordingly, the spontaneously emerging experiences of "in flow" and "not in flow" during the run were signaled through previously agreed, visible and distinguishable arm signs (right arm to indicate "in-flow" and left arm, "not in flow"). Participants were left free to report the "in flow" or "not in flow" state as many times they felt doing so. Each report was recorded with the corresponding time in which it happened. This procedure was identical to that used by previous authors [21], which was found to be more adequate than verbal reporting when fatigue accumulates [22].

\subsection{Data analysis}

The self-reported "in flow"/"not in flow" experiences while running were plotted for each participant to obtain velocity-dependent relations in the data series. Specifically, the series of each participant (i.e., time until test termination) were divided into five equal non-overlapping windows to obtain percentages of "in flow" in those windows:

Number of "in flow" reports in each time window $\times 100$

mber of total reports in each time window

The null hypothesis of a constant median over time was tested by means of a nonparametric repeated-measures Friedman analysis of variance (ANOVA). Wilcoxon matched-pairs test analysis was also performed to test statistically significant differences among the time windows (1st and 3rd; 1st and 5th; 3rd and 5th). Effect sizes (Cohen's d) were computed to demonstrate means' differences where effects approached $p<0.05$ level. The number of runners finishing "in flow" and those finishing "not in flow" was compared by using Chi square test. 


\section{Results}

\subsection{Manipulation check}

Participants reported a medium-high level of commitment to the running test $(M=7.21 \pm 2.04)$ and adhered strongly to the reporting procedures $(M=8.0 \pm 1.84)$. The perceived demand of the UMTT was rated as medium-high $(M=6.23, \mathrm{SD}=1.63$ of 9-point rating range). The running time was $M=23.03 \mathrm{~min}, S D=2.51$.

\subsection{Flow dynamics}

Fig. 1 displays four examples of individual time series where both "in flow" and "not in flow" reports are shown. The total number of reported changes oscillated among 11 and 1, respectively $(M=3.7 \pm 2.5)$. Participants alternated, following an idiosyncratic dynamic, the "in flow" and "not in flow" reports during the run, and completed the task following a "not in flow" report. The Chi square test revealed a significant effect, $\chi^{2}(N=20, d f=1)=9.82 ; p<.002$, indicating that more participants finished after reporting "not in flow" $(N=17 ; 85 \%)$ than "in flow" state $(N=3 ; 15 \%)$.

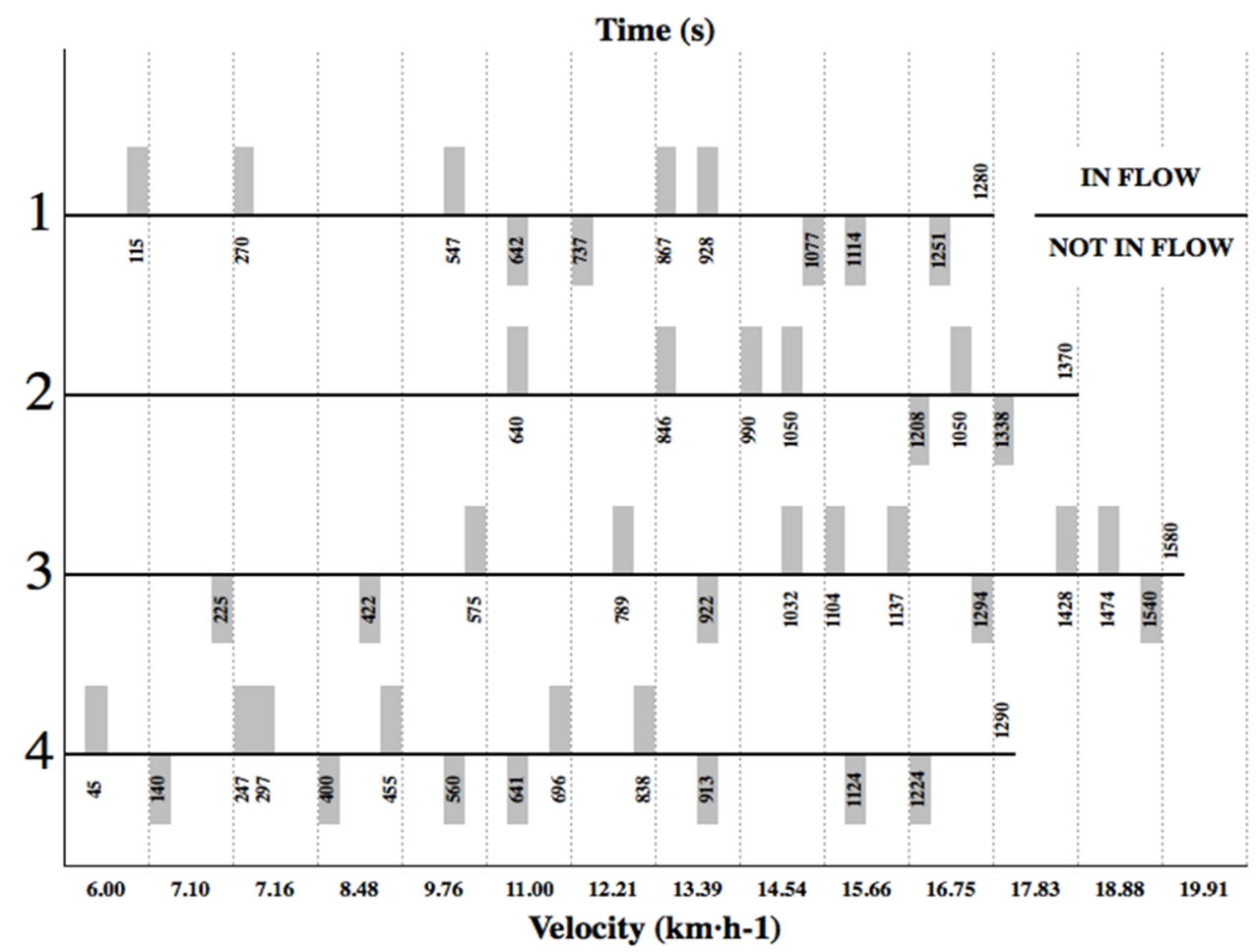

Fig. 1. Example of four typical individual time windows and velocity showing the dynamics of reporting "in flow" and "not in flow" states. Notice that the introspective reports lack magnitude. Prior to the point of voluntary exhaustion and effort termination, the "not in flow state" was significantly $(p<0.05)$ dominant

\subsection{Velocity-dependent "in flow" state}

Fig. 2 shows the median percentages of self-reported "in flow" states in the five velocity windows. The median started at $100 \%$ (1st window) and decreased to $84 \%$ and $50 \%$ in the second and third window, respectively. In the fourth window, however, it increased slightly to 
$67 \%$, to finally decrease abruptly to $25 \%$.

The Friedman ANOVA applied to the five intervals $(N=20, d f=4)$ revealed a significant effect, $\chi^{2}(20,4)=22.75, p<0.001$, of running velocity over the "in flow" experiences. Considering the "in flow" condition, the Wilcoxon test showed differences between the following intervals: 3rd and 5th $p<0.001$; 1st and 5th: $p<0.001)$ with Cohen's d coefficients: d(1st vs. 3 rd intervals $)=0.22$, d (1st vs. 5 th $)=1.35$, and $d(3$ rd vs. 5 th $)=1.28$.

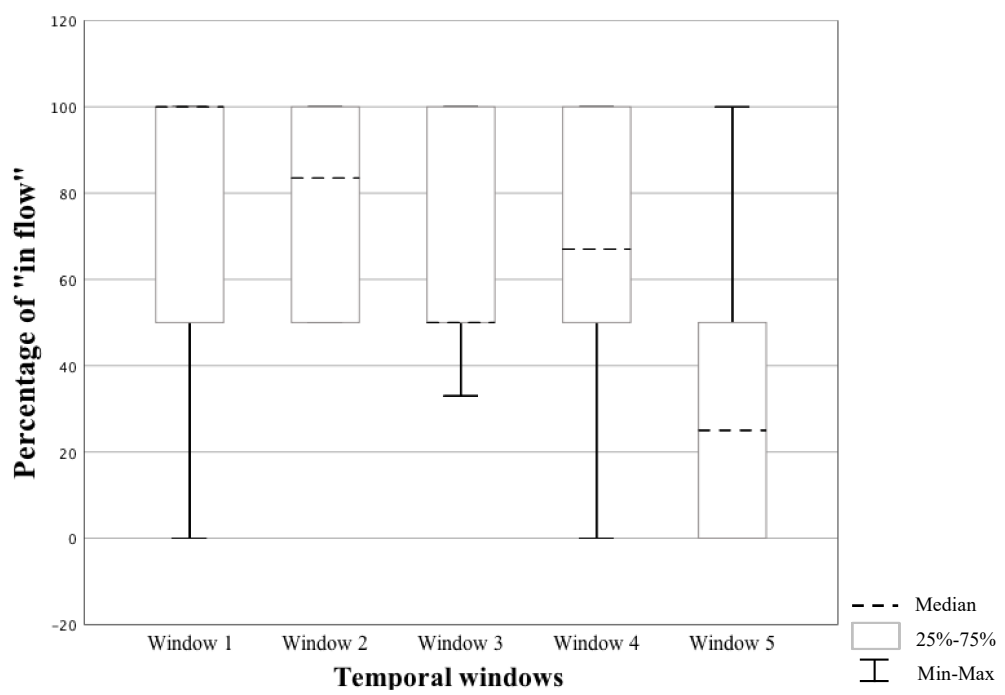

Fig. 2. Velocity-dependent median percentages of reporting "in flow" during the Université of Montréal Track Test

\section{Discussion}

Studying the dynamics of the flow experience during an incremental running velocity test revealed a metastable dynamic alternating the "in flow" and "not in flow" states, with an abrupt decrease of the "in flow" experience upon approaching voluntary exhaustion. The results also revealed unique flow pattern in the runners. Each one reported to be "in flow" and "not in flow" during different stages of the running task. Moreover, we noticed that the reports did not correspond to the regular velocity changes imposed by the running task protocol. These findings are supported by Swann et al.'s [8] argument that "flow seems to result from the interaction of internal states (e.g., focus, arousal, motivation, confidence, thoughts, and emotions), external factors (e.g., environmental and situational conditions) and behavioral factors (e.g., preparation)" (p. 813). As these boundary conditions were different among individuals and evolved at different time scales of observation [31], no identical flow dynamics were observed. Specifically, each runner maintained his/her own personal and environmental constraints, and each one of them evolved at their own pace. For example, and in line with the current findings, personal values and motivation evolve slowly compared to mood, affective valence and perceived effort [15].

The observed metastability of the flow state during the run in the current study reflected a relatively soft (i.e., flexible) coupling and integration between the involved component processes sensible to random perturbations. Specifically, small changes in perceived effort, pain, or fatigue could produce a change of the flow state report. In contrast, the stability of the "not in flow" state observed at the end of the running task reflected a weak coupling between the component processes and a small perturbation leading to voluntary exhaustion. The metastable dynamics, a subtle interval between both stability/instability extremes $[20,22,32]$, that seems to provide the very much needed flexibility of psychobiological processes was dominant during the run. Previous findings, which used introspective experiences, have revealed such metastable dynamics 
performing different types of exercise [16, 22, 33, 34].

The observed evolution of the median values of the "in flow" reports during the run indicates that the changes of personal constraints produced by the increasing velocity and effort accumulation affected the flow state. The dominant "in flow" experience in the first velocity window matched with Swann, Crust, and Vella's [35] findings, where athletes described being in flow from the start of the race. The results in the fourth velocity window, where percentages of "in flow" reports increased moderately from $50 \%$ to $67 \%$, can be related to the possibility of restoring or reinstating flow after disruption. In a previous study, $81 \%$ of athletes perceived it as possible to do so [36]. From the dynamic perspective, these state changes are attributed to the continuous creation/dilution of psychobiological synergies [15]. For example, in the case of attention allocation, the spontaneous reallocation of attention focus toward motivational task related thoughts compensates the power reduction of fatigued motor units [21]. The stabilization of every new synergy increases the efficiency of the system; the destabilization, in turn, results in the opposite effect.

Approaching the effort termination stage, the "not in flow" state was the dominant response reported by $75 \%$ of the runners. This abrupt change in the dynamics approaching exhaustion has been also found in previous studies investigating other psychobiological variables. As physical workload increases, attention allocation shifts from dissociation to association [13, 14, 22], affective responses shift negatively (displeasure) [1, 37], and at the point of termination, and shortly preceding it, participants were unable to form a new synergy to stabilize their perceived exertion shifts [16]. In fact, volitional exhaustion has been explained by the loss of stability mechanism $[38,32]$.

Two effort phases, metastable and stable (close to termination), respectively, have been also distinguished in constant and incremental power exercises performed until exhaustion when studying the dynamics of psychological variables $[13,14,16,17,22]$ and when testing a similar population in different environments [34], thereby confirming the robustness of the current findings.

Unlike the retrospective data collection strategy used in previous research on flow, this study proposed an online protocol, based on methodologies of cognitive science [27, 28, 39]. This methodology has been proved successful for capturing the dynamics of psychobiological variables such as attention focus [22], perceived exertion [21] and pain [17]. To the best of our knowledge, it is the first time that flow was self-monitored and self-reported during a physical task. The reason consists of the common believe that self-monitoring may impair the flow state. For instance, Csikszentmihalyi's [24] has stated that consciously being aware of flow during the activity is counterproductive and inhibits one's ability to merge action and awareness. In favor of self-monitoring the flow state on line, one can be argued that running is a simple cyclic type of activity and is less affected by the conscious awareness of flow. In addition, reporting through arm signs did not hinder the flow experience during the running test. From another perspective, the "loss of self-consciousness" was reported by less than $30 \%$ of the sampled athletes [8]; thus, questioning the specific flow dimensions and the relation between self-consciousness and the capacity to perceive flow. Nonetheless, in the literature it is already suggested that flow awareness is achievable [25].

The present findings share some practical implications. Instead of monitoring the training and competition workloads solely based on metabolic and physiological indices, the self-monitored flow method proposed herein may contribute to the accuracy of self-perception in the performer. Moreover, it can also prove to be a trustworthy tool for individualized monitoring safe practices [40]. Therefore, the flow dynamics may serve in recognizing the stability or instability profile of individual workloads. In sum, coaches can monitor psychophysiological states of athletes, help them identify individualized performance optimization strategies, and use biofeedback techniques to improve self-regulation. Also, recognizing the relation of flow dynamics and running pace can contribute to the non-elite runners' long-term engagement allowing to increase and control the running intrinsic rewarding experience. 
Concerning the study's limitations, participants in this study were young and active individuals. Consequently, caution is warranted when generalizing current findings to participants with different fitness levels. However, the consistency of the flow experience dynamics suggests that the qualitative results of this study may as well hold true for participants with different fitness levels. Also, running exercises with constant workload conditions may provide further insight into this concern.

By studying flow dynamics during incremental velocity running, a metastable behavior was revealed and an abrupt decrease of "in flow" experience upon approaching volitional exhaustion was evident. Self-monitoring flow experience can complement the use of physiological measures for a safe control of running velocity. Although its effectiveness must be further tested, the on-line self-monitoring of flow must be considered closely within performance-related research and application realms.

\section{Summary}

The dynamic of "flow experience" was investigated using an on-line self-monitoring method during an incremental velocity-running test performed until voluntary exhaustion. Results showed a meta-stable flow experience. The changes between "in flow" and "not in flow" states do not correspond to the imposed velocity changes. This finding points to flow as a complex, nonlinear process resulting from the performer-environment interaction. The online protocol proposed in this study can be used to capture the dynamics of flow, and could be also applied to self-monitor training and competition workloads, as well as for safe and individualized monitoring practices.

\section{Acknowledgements}

The authors would like to thank all who volunteered for the study and our colleagues Assumpta Ensenyat and Albert Cantón, from the University of Lleida (Spain), for their help in the experiments. No sources of funding were used to assist in the preparation of this manuscript.

\section{References}

[1] Ekkekakis P. Pleasure and displeasure from the body: perspectives from exercise. Cognition and Emotion, Vol. 17, 2003, p. 213-239.

[2] Dishman R. K., Sallis J. F., Orenstein D. R. The determinants of physical activity and exercise. Public Health Reports, Vol. 100, Issue 2, 1984, p. 158-171.

[3] Razon S., Hutchinson J. C., Tenenbaum G. Effort Perception. Measurement in Sport and Exercise Psychology, Champaign, Ill: Human Kinetics, 2012, p. 265-278.

[4] Csikszentmihalyi M. Beyond boredom and anxiety. San Francisco: Jossey-Bass. 1975.

[5] Csikszentmihalyi M. Flow: The Psychology of Optimal Experience. Harper \& Row, New York, Vol. 41, 1990.

[6] Schüler J., Brunner S. The rewarding effect of flow experience on performance in a marathon race. Psychology of Sport and Exercise, Vol. 10, Issue 1, 2009, p. 168-174.

[7] Stoll O. Peak Performance, the Runner's High, and Flow. Handbook of Sports and Exercise Psychology, American Psychology Association, Washington, Vol. 2, 2019, p. 447-465.

[8] Swann C., Keegan R. J., Piggott D., Crust L. A systematic review of the experience, occurrence, and controllability of flow states in elite sport. Psychology of Sport and Exercise, Vol. 13, Issue 6, 2012, p. 807-819.

[9] Montull Ll., Vázquez P., Rocas Ll., Hristovski R., Balagué N. Flow as an embodied state. Informed awareness of slackline walking. Frontiers in Psychology, Vol. 10, 2019, p. 2993.

[10] Turvey M. T., Kugler P. N. Chapter IVa an ecological approach to perception and action. Advances in Psychology, Vol. 17, 1984, p. 373-412.

[11] Shaw R. E., Kinsella-Shaw J. The survival value of informed awareness. Journal of Consciousnes Studies, Vol. 14, 2007, p. 137-154. 
[12] Ekkekakis P., Parfitt G., Petruzzello S. J. The pleasure and displeasure people feel when they exercise at different intensities: Decennial update and progress towards a tripartite rationale for exercise intensity prescription. Sports Medicine, Vol. 41, 2011, p. 641-671.

[13] Tenenbaum G., Connolly C. T. Attention allocation under varied workload and effort perception in rowers. Psychology of Sport and Exercise, Vol. 9, 2008, p. 704-717.

[14] Tenenbaum G. A Social-Cognitive Perspective of Perceived Exertion and Exertion Tolerance. Handbook of Sport Psychology. Wiley, New York, 2001, p. 810-820.

[15] Balagué N., Hristovski R., García-Retortillo S. Perceived Exertion-Dynamic Psychobiological Model of Exercise-Induced Fatigue. 4th ed., Handbook of Sport Psychology, Wiley, London, (in Press).

[16] Balagué N., Hristovski R., García S., Aragonés D., Razon S., Tenenbaum G. Intentional thought dynamics during exercise performed until volitional exhaustion. Journal of Sport Sciences, Vol. 33, Issue 1, 2015, p. 48-57.

[17] Slapsinskaite A., Hristovski R., Razon S., Balagué N., Tenenbaum G. Metastable pain-attention dynamics during incremental exhaustive exercise. Frontiers in Psychology, Vol. 7, 2017, p. 2054.

[18] Slapsinskaite A. Exercise-Induced Pain. Dynamic Perspective. Ph.D. Thesis, Universitat de Barcelona, Barcelona, Spain, 2017.

[19] Eyring H. The activated complex in chemical reactions. The Journal of Chemical Physics, Vol. 3, Issue 2,1935 , p. 107-115.

[20] Kelso J. A. S. Dynamic Patterns: the Self-Organisation of Brain and Behavior. MIT Press, Cambridge, 1995.

[21] Aragonés D., Balagué N., Hristovski R., Pol R., Tenenbaum G. Fluctuating dynamics of perceived exertion in constant-power exercise. Psychology of Sport and Exercise, Vol. 14, 2013, p. 796-803.

[22] Balagué N., Hristovski R., Aragonés D., Tenenbaum G. Nonlinear model of attention focus during accumulated effort. Psychology of Sport and Exercise, Vol. 13, 2012, p. 591-597.

[23] Hristovski R., Balagué N., Schöllhorn W. Basic Notions in the Science of Complex Systems and Nonlinear Dynamics. Complex Systems in Sport (p. 3-17). Routledge, London, 2014.

[24] Csikszentmihalyi M. Finding Flow: the Psychology of Engagement with Everyday Life. Harper Perennial, New York, 1997.

[25] Swann C., Piggott D., Crust L., Keegan R., Hemmings B. Exploring the interactions underlying flow states: A connecting analysis of flow occurrence in European Tour golfers. Psychology of Sport and Exercise, Vol. 16, 2015, p. 60-69.

[26] Leger L., Boucher R. An indirect continuous running multistage field test: University of Montreal track test. Canadian Journal of Applied Sports Sciences, Vol. 5, 1980, p. 77-84.

[27] Giambra L. M. Task-unrelated thought frequency as a function of age: a laboratory study. Psychology and Aging, Vol. 4, 1989, p. 136-143.

[28] Giambra L. M. A laboratory method for investigating influences on switching attention to taskunrelated imagery and thought. Consciousness and Cognition, Vol. 4, 1995, p. 1-21.

[29] García T., Jiménez R., Santos-Rosa J., Reina R., Cervelló E. Psychometric Properties of the Spanish Version of the Flow State Scale. The Spanish Journal of Psychology, Vol. 11, Issue 2, 2008, p. $660-669$.

[30] Jackson S., Marsh H. Development and validation of a scale to measure optimal experience: The Flow State Scale. Journal of Sport \& Exercise Psychology, Vol. 18, 1996, p. 17-35.

[31] Papo D. Time scales in cognitive neuroscience. Frontiers in Physiology, Vol. 4, Issue 86, 2013, p. 1-10.

[32] Hristovski R., Balagué N. Fatigue-induced spontaneous termination point -Nonequilibrium phase transitions and critical behavior in quasi-isometric exertion. Human Movement Science, Vol. 29, 2010, p. $483-493$.

[33] García S., Razon S., Hristovski R., Balagué N., Tenenbaum G. Dynamic stability of task-related thoughts in trained runners. The Sport Psychologist, Vol. 29, 2015, p. 302-309.

[34] Slapsinskaite A., García S., Razon S., Balagué N., Hristovski R., Tenenbaum G. Cycling outdoors facilitates external thoughts and endurance. Psychology of Sport and Exercise, Vol. 27, 2016, p. 78-84.

[35] Swann C., Crust L., Vella S. New directions in the psychology of optimal performance in sport: flow and clutch states. Current Opinion in Psychology, Vol. 16, 2017, p. 48-53.

[36] Chavez E. Flow in sport: A study of college athletes. Imagination, Cognition and Personality, Vol. 28, 2008, p. 69-91. 
[37] Sheppard K. E., Parfitt G. Patterning of physiological and affective responses during graded exercise test in sedentary men and boys. Journal of Exercise Science and Fitness, Vol. 6, Issue 2, 2008, p. 121-129.

[38] Balagué N., Torrents C., Hristovski R., Davids K., Araújo D. Overview of complex systems in sport. Journal of Systems Science and Complexity, Vol. 26, Issue 1, 2013, p. 4-13.

[39] Smallwood J., Schooler J. The restless mind. Psychological Bulletin, Vol. 132, 2006, p. 946-958.

[40] Balagué N., Hristovski R., Almarcha M. C., García-Retortillo S., Ivanov P. Network physiology of exercise: vision and perspectives. Frontiers in Physiology, Vol. 11, 2020, p. 611550.

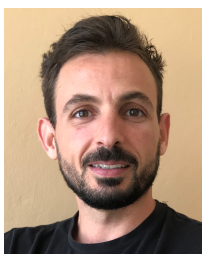

Aleix Gibert is a Ph.D. student in Sports Science at University of Lleida. He works at the University of TecnoCampus, Mataró, and at FC Barcelona. His current research interests include flow state and mindfulness. He is part of the Complex Systems in Sport research group.

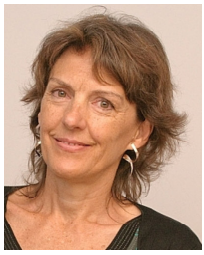

Natàlia Balagué received Ph.D. degree in Sports Science from University of Barcelona, Spain, in 1990. She is Professor of Exercise Physiology at INEFC, Universitat de Barcelona, and leads the Complex Systems in Sport research group. Her current research interests include network physiology of exercise and complex systems in sport.

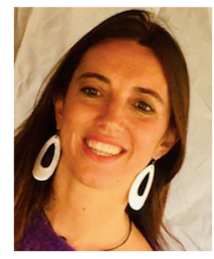

Carlota Torrents received Ph.D. degree in Sports Science from University of Barcelona, Spain, in 2005. She is Professor in University of Lleida and she is part of the Complex System and Sport research group. Her current research interests include creativity and collective motor behavior.

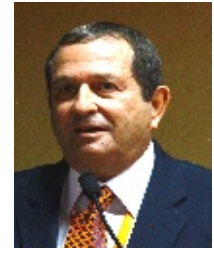

Gershon Tenenbaum was the Benjamin S. Bloom Professor of Educational and Sport Psychology and the Sport Psychology Graduate Program Director at Florida State University. Currently he is a Professor and Head of the Graduate Program in Sport Psychology in the Interdisciplinary Center, School of Psychology, Herzliya, Israel and teaches sport psychology in the Department of Psychology, the College of Management in Rishon Letsion, Israel. He is a graduate of Tel-Aviv University and the University of Chicago in measurement and research methods in psychology. 